\title{
A Dificuldade de Aceitação no Processo Saúde e Doença Diante o Diagnóstico Renal Crônico: A Importância do Psicólogo
}

\author{
The Difficulty of Acceptance in the Health and Disease Process Before Chronic Kidney Diagnosis: \\ The Importance of the Psychologist
}

Rosimar Viana Baptista Correa ${ }^{\dagger}$, Barbara Silveira ${ }^{\ddagger}$

Como citar esse artigo. Correa, R.V.B.; Silveira, B. A Dificuldade de Aceitação no Processo Saúde e Doença Diante o Diagnóstico Renal Crônico: A Importância do Psicólogo. Revista Mosaico - 2019 Jul/Dez.; 10 (2): SUPLEMENTO 32-39

\section{Resumo}

Entre os acometimentos crônicos a doença renal crônica é uma das mais agressivas tanto em aspectos físicos, quanto em aspectos emocionais e sociais, pois, consiste na perda progressiva e irreversível da função renal, e da necessidade de terapia renal substitutiva, onde existe uma indumentária tecnológica no qual o portador precisar manter-se conectado por horas do dia, para a manutenção da vida. O objetivo do presente trabalho foi evidenciar a atuação do psicólogo com o paciente renal crônico, e possui como especificidade: evidenciar as dificuldades de aceitação do paciente renal crônico; descrever as rotinas de psicologia para assistir o paciente no processo de doença renal crônica; evidenciar a intervenção de psicologia nas terapias renais substitutivas. Para isso utilizou como método a revisão bibliográfica integrativa, e concluiu que o profissional psicólogo possui importância grandiosa no que tange a aceitação do quadro de doença renal crônica pelo paciente, e também, atua com a equipe e com a família, possuindo então uma função valiosíssima de aproximação e empatia, gerando assim um elo entre o paciente renal crônico e todos os membros que fazem parte de sua rotina diária.

Palavras-chave: Psicologia, aceitação, doença renal crônica.

\begin{abstract}
Among chronic diseases, chronic renal disease is one of the most aggressive in both physical and emotional and social aspects, since it consists of progressive and irreversible loss of renal function, and the need for renal replacement therapy, where there is a technological which the bearer needs to keep connected for hours of the day, for the maintenance of life. The objective of the present study is to demonstrate the performance of the psychologist with the chronic renal patient, and has as its specificity: evidence of the difficulties of acceptance of the chronic renal patient; describe the psychology routines to assist the patient in the process of chronic kidney disease; psychology intervention in renal replacement therapies. In order to do so, he used the integrative bibliographic review as a method, and concluded that the professional psychologist has great importance in regard to the patient's acceptance of chronic kidney disease, and also works with the team and the family, having a very valuable function of rapprochement and empathy, thus generating a link between the chronic renal patient and all the members that are part of their daily routine.
\end{abstract}

Keywords: Psychology, acceptance, chronic kidney disease.

\section{Introdução}

A expectativa de todo ser humano é que sua vida possua prosperidade, que seja longínqua e satisfatória, e adoecer e todo o processo que permeia o evento não faz parte dos planos. Todo o processo diagnóstico de uma doença gera conturbações psicossomáticas capazes de definir os rumos que essa moléstia irá tomar, incluindo passos do tratamento, a qual médico e especialidade buscam para o acompanhamento, as hipóteses de sucesso terapêutico, entre outros. Todo esse aparato sentimental e de atitudes são potencializados quando a descoberta gira em torno de uma doença crônica (PACHECO; SANTOS; BREGMAN, 2006).

As doenças de caráter crônico vêm progressivamente aumentando na população, não considerando nenhum aspecto étnico, social, racial tampouco etário, de forma exponencial ao redor de todo mundo, e são definidas como uma doença permanente ou de longa duração, em geral, com lenta progressão, que pode ou não produzir, incapacidade ou deficiência, causada por patologias irreversíveis, que necessitam de reabilitação ou aparatos para manter a vida (NASCIMENTO, 2013).

Entre os acometimentos crônicos a doença renal crônica é uma das mais agressivas tanto em aspectos físicos, quanto em aspectos emocionais e sociais, pois, consiste na perda progressiva e irreversível da função renal, e da necessidade de terapia renal substitutiva, onde existe uma indumentária tecnológica no qual o portador precisa manter-se conectado por horas do dia, para a manutenção da vida (CAMPOS; TURATO, 2010).

Afiliação dos autores: † Psicóloga, graduado pela Universidade de Vassouras; pós-graduando em Psicologia Hospitalar e da Saúde pela Universidade de Vassouras (Vassouras-RJ, Brasil).

¥ Psicóloga, Mestre em Ensino de Ciências da Saúde - Universidade Federal de São Paulo (SP); Especialista em Psicanálise; Professora de Pós-Graduação em Psicologia Hospitalar e da Saúde na Universidade de Vassouras, Vassouras-RJ, Brasil; Supervisora da Residência em Psicologia Hospitalar no HUV (Hospital Universitário de vassouras), pela Universidade de Vassouras. 
As terapias renais substitutivas são empregadas com a função de remover líquidos e produtos residuais da urina do organismo, necessitando de adaptação do paciente a forma dialítica empregada, assim como cuidados exacerbados com higiene e cuidados corporais como forma profilática de evitar processos infecciosos que são muito nocivos aos pacientes (ROMÃO JR, 2004).

A adesão ao plano de tratamento não deve ser realizada apenas com o intuito de manutenção da vida, visto que não será uma aceitação e sim apenas uma adequação com a finalidade de não morrer, pois, a primeira opção gira em torno de mudança de hábitos e estilo de vida, com melhor e mais amplo prognostico, maior sobrevida e qualidade de vida, o segundo gira em torno de declínio, de degeneração progressiva e prognóstico sombrio (RESENDE et al., 2007).

É de extrema importância a reconstrução de todos os agentes psíquicos do paciente renal crônico para o convívio social, pois, o quadro sintomatológico apresentado pelo paciente não é apenas uma doença, é um fenômeno de impacto vital significativo, que incidirá nos hábitos e na rotina da pessoa de forma drástica, podendo associar-se a vários outros fatores orgânicos e psicossomáticos, possibilitando a não adesão ao plano de tratamento e em muitos casos a morte (DINIZ; SCHOR, 2006).

A equipe multidisciplinar precisa estar coesa para a elaboração de um plano de tratamento capaz de suprir as necessidades do paciente renal crônico e também estar apta a educar e amparar frente ao evento, com a finalidade de gerar no paciente uma aceitação verdadeira de sua morbidade, uma reabilitação mais rápida e menores danos oriundos do agravo. Portanto, o paciente renal crônico e suas dificuldades de aceitação do plano de tratamento e da própria doença é o objeto do presente estudo, que se justifica à importância e os desafios que o mesmo enfrenta no evento.

O objetivo do presente trabalho evidencia a atuação do psicólogo com o paciente renal crônico e como especificidade: evidenciar as dificuldades de aceitação do paciente renal crônico; descrever as rotinas de Psicologia para assistir o paciente no processo de doença renal crônica; evidenciar a intervenção de Psicologia nas terapias renais substitutivas.

\section{Metodologia}

Trata-se de uma revisão integrativa sobre assistência de Psicologia ao paciente renal crônico. A Pesquisa foi efetuada na Biblioteca Virtual BIREME, com documentação indireta nos bancos de dados Medline, LILACS, sendo utilizados os descritores, doença renal crônica, aceitação e psicologia, com o conector boleano "and".
A busca ocorreu no mês de janeiro, tendo sido incluídos artigos em português com recorte temporal de 2010 a 2018.

\section{Revisão de Literatura}

\section{A doença renal crônica}

Os rins são órgãos fundamentais para a manutenção da homeostase do corpo humano, por essa razão que a diminuição da função renal, implique em comprometimento de essencialmente todos os outros órgãos. A doença renal crônica é uma lesão presente por um período igual ou superior a três meses, definida por anormalidades estruturais ou funcionais do rim, com ou sem diminuição da filtração glomerular, evidenciada por anormalidades histopatológicas ou de marcadores de lesão renal, incluindo alterações sanguíneas ou urinárias, ou ainda de exames de imagem (REIS; GUIRARDELLO; CAMPOS, 2008).

Existem grupos de indivíduos que são mais suscetíveis à doença renal crônica portanto, são considerados grupos de risco, tais como: Hipertensos, Diabéticos, idosos, pacientes com doença cardiovascular, familiares de pacientes portadores de doenças renais crônicas e pacientes em uso de medicamentos nefrotóxicos (SILVA; THOMÉ, 2009).

A avaliação diagnóstica mais precisa para a identificação fidedigna da doença renal crônica é a filtração glomerular, onde o nível da mesma sofre variação com a idade, sexo, e massa muscular. Mediado pela incapacidade de surtir efeito nos tratamentos conservadores, nos déficits nutritivos, e/ou complicações relacionadas a uremia, são os melhores indicativos de terapia renal substitutiva (NASCIMENTO, 2013).

As modalidades dialíticas devem ser consideradas no plano de assistência do cliente, visto que, o melhor tratamento influi grandiosamente na vida do cliente, expõe-se que a diálise peritoneal é um método mais simples, com índice maior de suavidade e evita desequilíbrios abruptos, contudo, é ineficaz quando existem riscos decorrentes da necessidade de anticoagulação. Todavia, a hemodiálise é rapidamente instituída, com a instalação de um cateter em veia central ou por via de fístula arteriovenosa, e é mais indicada em casos de hipercalcêmica ou distúrbios eletrolíticos. Contudo, deve-se iniciar de caráter lento, de forma mais curta e frequente, pois, por possuir maior eficácia, existe o risco da síndrome do desequilíbrio da diálise (MALDANER et al., 2008).

Pacientes com doença renal crônica apresentam alterações importantes na fisiologia renal, na vascularização e nos fatores cardiológicos, que devem ser corrigidos e cuidados de forma eficaz, pois, podem 
ocasionar danos extensos, inclusive a morte, podendo apresentar em seu quadro clínico anemias, hipertrofia ventricular esquerda, síndrome cardiorrenal, calcificação cardiovascular, entre outros danos. (SILVA \& THOMÉ, 2009).

O diagnóstico e tratamento precoce em qualquer forma de lesão renal seja ela de caráter agudo ou crônico é de suma importância para um melhor prognóstico, para uma maior viabilidade de planos terapêuticos e para a melhoria na qualidade de vida. Vale ressaltar que a prevenção nos grupos mais suscetíveis ainda é uma das ferramentas mais essenciais para o não surgimento de doenças renais, ou para que seus efeitos sejam atenuados.

\section{A terapia renal substitutiva}

Inúmeros avanços surgiram nos serviços dialíticos desde seu surgimento, seja no âmbito de conhecimento científico, seja no que diz respeito às novas tecnologias. No início do processo o mesmo era realizado em sua maioria pela equipe médica, depois, com o aprimoramento da técnica e a expansão do trabalho, outros profissionais foram agregando-se a equipe multidisciplinar, e hoje, toda a equipe de saúde atua em Nefrologia (BARROS, 2006).

Depois de tal dissertação evidencia-se a importância da qualificação e do aprimoramento constante do conhecimento por parte do profissional que atua em Nefrologia, visto à grande complexidade inerente a área, consiste a diálise em um procedimento que por intermédio de um acesso, seja ele peritoneal ou venoso, permite um fluir do sangue ou do líquido excedente do organismo, de maneira elevada, sendo esse transportado por um sistema de circulação de modo extra corporal até um filtro, onde será logo após ser processado, devolvido ao corpo (SOUSA, 2013).

A maioria dos pacientes que são submetidos a terapia renal substitutiva está em uma clínica ou dentro de um hospital, em geral, obedecendo a uma prescrição que é seguida no que diz respeito a frequência e duração das sessões, podendo ser convencionado em um esquema regular ou não (BARROS, 2006).

Barros et al. (2006) trazem uma definição envolvendo os aspectos relacionados um pouco mais com os equipamentos utilizados e com as manifestações fisiológicas que envolvem o paciente, dos quais compreende um circuito que atua de maneira extra corporal, participando do processo o filtro ou dialisador, que é um aparato formado por fibras ocas dispostas paralelamente, das quais essas fibras possuem poros, onde a água é eliminada com os solutos de baixo e médio peso molecular. Dentro desse processo o sangue ou os fluidos corporais são transportados através de um acesso, podendo ser por um cateter em uma veia, uma fístula arteriovenosa, uma prótese ou um cateter peritoneal, impulsionada por uma bomba para um sistema, o dialisador. Essa difusão dos solutos entre o sangue/fluidos corporais e a solução da diálise possui como resultado a remoção dos resíduos nocivos do sangue e a reposição de alguns outros que possam ser necessários.

Riella et al. (2010) entende que a unidade de diálise deve ser planejada para que o paciente renal crônico receba todo o suporte necessário para o seu tratamento com as melhores condições possíveis como, materiais, equipamentos, área física e acima de tudo cuidados, com profissionais bem especializados, com excelência técnica e com atendimento humanizado, com um ambiente com boa condição para a equipe que nela atua.

\section{O paciente renal crônico}

Todo processo de enfermidade, mesmo as mais brandas e pouco nocivas ao organismo, gera fragilidade e vulnerabilidade, seja orgânica, fisiológica e/ou emocional, com mudanças consideráveis na vida do indivíduo, isso se torna mais exacerbado frente a uma doença crônica visto que ocorrem alterações na rotina diária do paciente, costumes, alterações de caráter físico/morfológico e também, psicológicas (CAIUBY; KARAM, 2010).

Uma das doenças crônicas com caráter mais agressivo, prejudicial e com altos índices de mortalidade é a renal, que compreende a perda progressiva e irreversível das funções dos rins, criando assim, um déficit significativo na homeostase orgânica, e gerando complicações sistêmicas grandiosas (SOCIEDADE BRASILEIRA DE NEFROLOGIA, 2014).

Os tratamentos dialíticos podem ser conduzidos de muitas formas, contudo, não possuem uma cura, mesmo com o advento do transplante renal, sendo este mais um dos variados tratamentos, ou o tratamento conservador, a diálise peritoneal e a hemodiálise. Mesmo com toda relevância terapêutica, para a manutenção da vida e a busca pela normalidade do equilíbrio orgânico, os tratamentos são bastante agressivos, monótonos e em certos momentos, dolorosos (GUYTON; HALL, 2006).

Várias restrições são impostas ao paciente portador de doença renal crônica, seja em âmbito alimentar, de ingestão hídrica e em muitos casos até de circulação, causando no paciente uma mudança onerosa de rotina, importantes danos aos aspectos psicossociais e emocionais, visto a implementação de uma rotina diária para que a vida possa ser mantida (NAKAO, 2013).

O sofrimento imposto ao paciente, seja no processo de adoecimento ou tratamento, causam eventos adversos como depressão e ansiedade, além 
de comprometimentos no ego, de déficit na autoestima, disfunções sexuais e síndromes psico-orgânicas, sendo originados na maioria das vezes por várias perdas sofridas pelo paciente, a da saúde, de identidade, das condições de trabalho, do autodomínio e também o medo do desconhecido, este último muitas vezes gera não aceitação/adesão do plano terapêutico implementado, e evasão dos serviços dialíticos preconizados (CAIUBY; KARAM, 2010).

O paciente renal crônico, mesmo com todas as imposições e restrições causadas pela doença em comum acordo com o plano de tratamento, busca a compreensão e aceitação da sua doença, visando identificar a origem e a terapêutica que será instaurada. Contudo, o processo não é fácil, gerando uma exacerbação de dúvidas e medos, capazes de gerar, no paciente, dependência familiar em demasia, falha na auto imagem, na autonomia e na liberdade, gerando a necessidade do profissional psicólogo para atuar com essa clientela (SIMONE, 2011).

\section{A Psicólogia e o Paciente renal crônico}

A Psicologia que atua em Nefrologia vislumbra compreender as imposições causadas pelo tratamento e também às limitações da própria doença, e idealizar o paciente como um ser participante da sociedade, trabalhando com a intenção de reabilitação com intuito de minimizar o sofrimento do processo saúde-doença (STRAUB, 2014).

$\mathrm{O}$ resgate da essência e a busca pela justificativa do estado de saúde atual do paciente são os objetos fundamentais que o psicólogo hospitalar que atua em Nefrologia visa resgatar, com a intenção primordial de avaliar cada queixa apresentada pelo paciente, com o pensamento sempre amplo em todos os aspectos e sinais apresentados pelo mesmo, no enfrentamento e aceitação (FARIAS, 2012).

O paciente renal crônico possui associado ao seu plano de tratamento um alto risco de eventos adversos, incluindo a própria morte, devido a instabilidades orgânicas variadas, as falências de órgãos associados à função renal, tais como os pulmões e o coração, entre outros, e o psicólogo precisa saber lidar com essa gama de adversidades apresentadas, pois, como cada indivíduo reage de forma imprevisível frente às situações diversas (TORRES, 1999).

A compreensão da dor, a postura de cada indivíduo que está adoentado e o respeito por cada apresentação e manifestação álgica é essencial para um atendimento de qualidade de psicologia nefrologista, sempre se munindo da escuta terapêutica colaborativa, sem opressão, apenas permitindo ao paciente que se comunique e relate seus medos e anseios quando o mesmo julgar necessário, contudo, sempre evidenciando a liberdade para que ele possa falar de si mesmo no momento que crer ser oportuno (MUCILLO, 2006).

O psicólogo fundamenta-se no apoio ao indivíduo doente, para buscar novos significados para a vida do mesmo, facilitando assim a comunicação, o enfrentamento, evitando a evasão terapêutica, proporcionando o cuidado, fechando feridas abertas pelo tempo e possibilitando formas de conversar sobre o adoecer e sobre a finitude de uma das suas funções orgânicas, sem sentir-se agredido (MACIEL, 2006).

O paciente ao receber a notícia de que se tornou portador de doença renal crônica passa a questionar a própria existência, suas crenças e religiosidade, assumindo, portanto, uma personalidade defensiva, como se o quadro fosse uma forma punitiva por erros ou por algum demérito enquanto era saudável. Todas essas perdas culminam em busca por justificativas e tentativas de compreensão, girando em estágios de negação, raiva, barganha, aceitação e esperança (KUBLER-ROSS, 2011).

Acolher a família do paciente é fundamental, pois, ela participa de todo o processo junto, compartilha as dores, anseios, sente-se incapaz tecnicamente de ajudar ou somar com o tratamento, muitas vezes sentem-se culpados pelo agravamento do quadro e pela cronicidade da doença instaurada, portanto, o profissional psicólogo deve escutar também a família, perceber sua dor em descobrir que a doença renal não tem cura, todavia, evidenciar e implementar a esperança de que mesmo com o tratamento agressivo e invasivo, ainda existe vida e formas de cuidado e qualidade, além de revelando a possibilidade de não ter mais o que fazer (MACIEL, 2006).

\section{Análise e Discussão dos resultados}

A doença renal crônica é um evento muito traumático aos indivíduos que a portam, pois a mesma compromete além da homeostasia orgânica, toda a construção psicossocial do indivíduo, sendo uma das doenças crônicas de mais difícil manutenção, com índices altíssimos de mortalidade, associada ao crescimento anual exponencial.

A Psicologia atua em todo processo durante o evento, seja no momento do diagnóstico, seja na aceitação do plano terapêutico implementado, seja no suporte ao cliente dialítico e à família, no controle emocional da equipe e no enfrentamento junto ao paciente de seu quadro de saúde.

Frenteànecessidadede atualizaçãoconstante, visto que a área de Nefrologia denota em si aprimoramento de caráter permanente, o presente estudo vislumbrou a busca por artigos recentes, com o corte temporal de oito anos, e analisou que em 2010 foi publicado um artigo sobre o assunto (16,6\%), assim como em 2012 
foi publicado um artigo sobre o assunto (16,6\%), seguido por 2014 com um artigo publicado $(16,6 \%)$, 2016 com um artigo publicado $(16,6 \%)$ e 2017 com dois artigos publicados $(33,6 \%)$. Analisando esse resultado, é demonstrado um aumento de pesquisas relacionadas ao tema, até os dias atuais, portanto, deixa explícita a preocupação literária com os pacientes renais crônicos e sua aceitação. O gráfico 1 evidencia essa afirmativa.
Os variados tipos de estudo influenciam muito no formato das pesquisas e na forma com que elas podem ser analisadas, evidenciando as formas de busca que os autores fizeram e os mecanismos que os levaram a dissertar suas conclusões. O presente trabalho evidenciou que apenas um artigo dentre todos analisados era do formato revisão bibliográfica $(16,6 \%)$ e cinco eram descritivo-exploratório $(83,4 \%)$. O gráfico 2 segue com essa afirmativa em porcentagem.

Gráfico 1. Porcentagem referente ao ano em que foram publicados os artigos

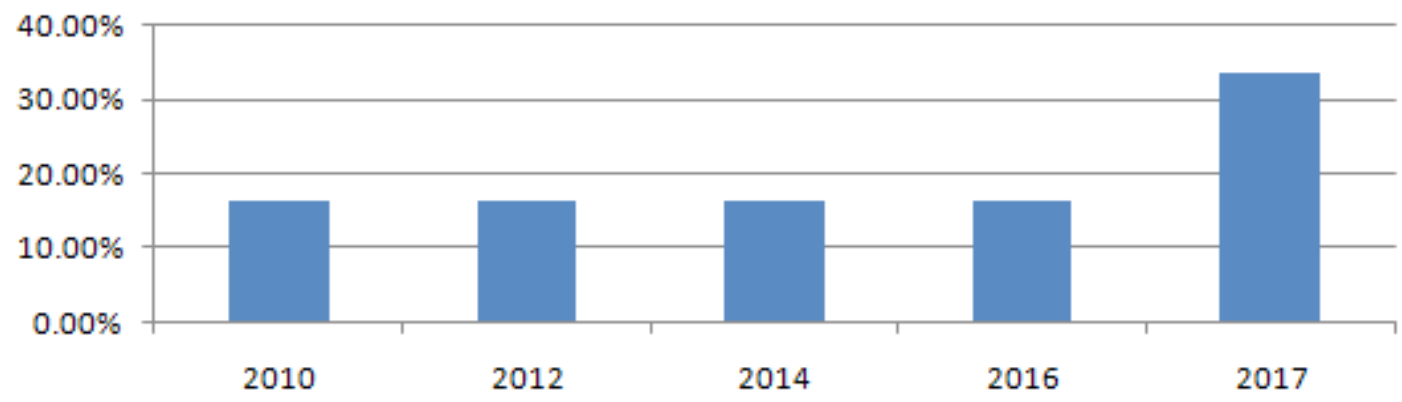

Gráfico 2. Porcentagem referente ao tipo de estudo encontrado na análise

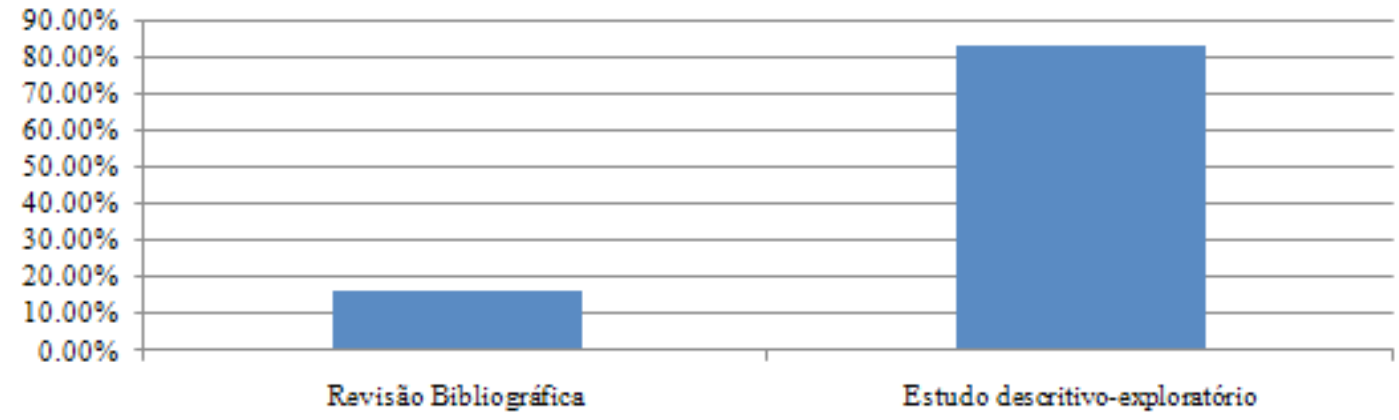

A dificuldade no processo de aceitação da doença renal crônica é um evento muito rotineiro nos serviços de nefrologia, pois, é notável que visualizada a perda de uma das funções mais primordiais do organismo, o indivíduo rejeite em primeiro instante estar conectado a um equipamento para manter-se vivo. Frente a essa afirmativa o presente estudo verificou a forma como os trabalhos estudados abordam a dificuldade de aceitação da doença renal crônica, concluindo que dois artigos abordam indiretamente o processo $(33,2 \%)$ e quatro artigos abordaram diretamente o assunto $(66,8 \%)$. O gráfico 3 demonstra essa afirmativa:

A compreensão da atuação do profissional psicólogo é um dos objetivos do presente trabalho com o intuito de evidenciar a atuação desse sujeito da equipe multidisciplinar atuante em Nefrologia, elaborou-se o quadro 1 evidenciando todo o plano terapêutico do psicólogo frente ao paciente portador de doença renal crônica. 
Gráfico 3. Porcentagem referente a forma como os estudos abordam a aceitação da doença renal crônica

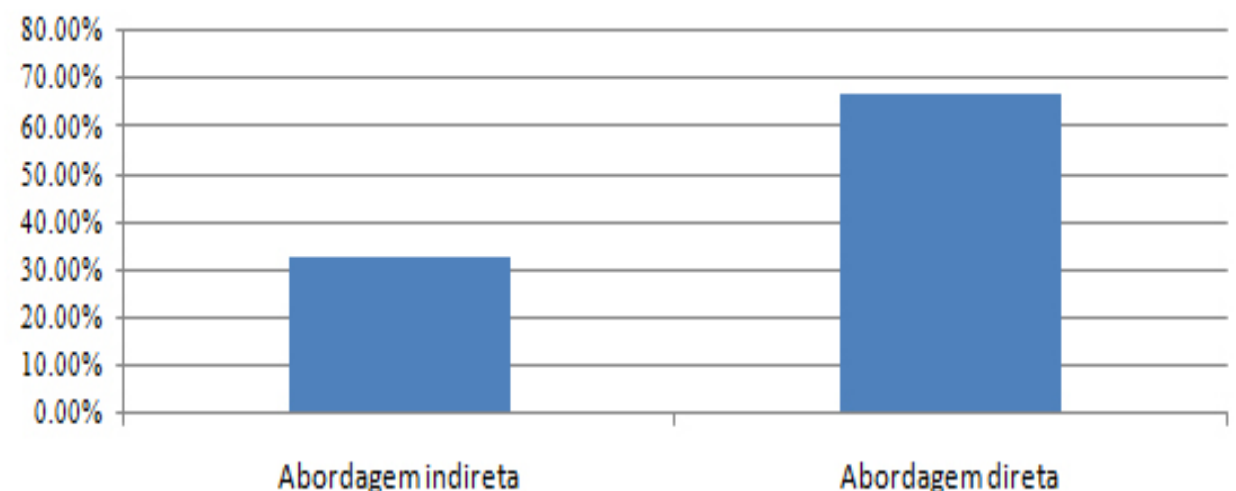

Quadro 1. Caracterização da atuação do psicólogo nefrologista frente ao cliente renal crônico

Promover a integração do paciente, da família e da equipe;

Favorecer a interação para a criação de um ambiente mais positivo para o tratamento;

Orientar o paciente sobre o tratamento dentro do âmbito psicológico e as consequências

da não-adesão;

Acompanhar individualmente cada paciente coma finalidade de detectar fragilidades e possíveis mecanismos que possam comprometer o plano de assistência;

Orientar e realizar a harmonização do paciente com a equipe multidisciplinar, gerando a feto mútuo;

Acompanhar rotineiramente o avanço ou involução do qua dro emocional do paciente; Orientar a familia quanto às mudanças que o paciente irá demonstrar, tanto física, quanto psicologicamente;

Acompanhar a familia do paciente coma finalidade de compreender o meio em que o mesmo vive vislumbrando detectar fragilidades capazes de comprometer o plano de assistência;

Instaurar grupos terapêuticos com a finalidade de manejar as emoções e facilitar o processo de aceitação;

Identificar problemáticas emocionais coletivas e individuais, assim como encontrar soluções que possam potencializar o plano de assistência;

Criar reflexões sobre o "estar vivo", gerando assim auxilio na superação, no Elaborar trabalhos coletivos que possuam como finalidade a divisão de dúvidas, angústias e receios, e buscando formas de sanar individualmente cada uma;

Oferecer meios de autocontrole e manejo de emoções, para que o paciente possa reconhecer seus momentos de alegrias e frustrações frente ao tratamento, e assim, gerar confiança, aceitação e esperança. 
O processo de descoberta da doença renal crônica gera uma avalanche de sentimentos que tornam dificultosa a adesão, e fazem com que haja uma ruptura do padrão de vida que outrora era vigente, perdendo o sentido da vida. É uma sensação pesarosa vivenciada como luto, devido à perda incurável de uma função orgânica, envolvendo cinco estágios distintos e não subsequentes, sendo eles a negação e isolamento, que consiste na grande dificuldade de compreender sua nova condição, desencadeando pensamentos negativos em relação a si e ao mundo, buscando a todo tempo, reclusão, demonstrando a fuga da realidade. $\mathrm{O}$ segundo estágio consiste na raiva, outrora o sentimento que era oculto, recluso, frente a vivência de vários traumas torna-se exteriorizado, com atitudes de revolta e certa agressividade. $\mathrm{O}$ terceiro estágio é a barganha, onde o paciente portador de doença renal crônica busca um tipo de acordo, de refúgio, capaz de mudar sua condição e destino, é uma criação de uma fantasia, algo irreal, onde a carga se torna mais leve. Quarto estágio é a depressão, caracterizada pela compreensão total da perda e o choque frente à dependência de um indumentário tecnológico para a manutenção da vida, provocando junto ao luto e ao pesar, sentimento de impotência e angústia. Quinto estágio é a aceitação, onde o paciente portador de doença renal crônica aceita sua condição de ser no mundo, passando, portanto, por enfrentamentos de suas limitações e adquirindo consciências sobre as possibilidades de vida (KUBLER-ROSS, 2011).

$O$ presente trabalho encontrou na literatura estudada, agentes que são cruciais para a dificuldade na aceitação da doença renal crônica, os mesmos foram elencados no quadro 2.

Quadro 2. Caracterização dos agentes complicadores na adesão e aceitação da doença renal crônica (DRC)

\author{
A juventude e a sensação de "vida inteira pela frente"; \\ Negação e o discurso de "eu não me acho doente"; \\ Mudanças na estética corporal e a sensação de "sentir-se feio"; \\ Disfunções sexuais e a sensação de "impotência e ausência de virilidade" \\ Medo do desconhecido e a sensação de "irei morrer" \\ Perda da liberdade e sensação de "depender de uma máquina para viver" \\ Conformismo e o discurso de "todos irão morrer um dia"
}

Maturana et. al, 2016.

\section{Considerações Finais}

O estudo evidenciou a atuação do psicólogo e analisou as dificuldades de aceitação do paciente renal crônico; expondo as rotinas de Psicologia para assistir o paciente no processo de doença renal crônica e a intervenção de Psicologia nas terapias renais substitutivas.

Verificou-se que o profissional psicólogo possui importância grandiosa no que tange a aceitação do quadro de doença renal crônica pelo paciente, e a atuação com a equipe e família, possuindo então uma função valiosíssima de aproximação e empatia, gerando assim um elo entre o paciente renal crônico e todos os membros que fazem parte de sua rotina diária.

No campo de Nefrologia, situações surgem para confrontar o paciente, tornando-o um indivíduo cheio de dúvidas, medos, anseios e conflitos. O profissional psicólogo com a escuta terapêutica, na prática de atendimento individualizado e coletivo, oferta apoio ao paciente, munindo-o de educação sobre a sua nova condição.

Vale ressaltar que o número reduzido de publicações sobre o tema abordado faz com que a pesquisa limite-se, portanto se torna necessário que o profissional de Psicologia que atua em Nefrologia exponha suas pesquisas e vivências para que mais trabalhos possam ser construídos. 


\section{Referências}

BARROS, E. et. al.. Nefrologia: Rotinas, diagnóstico e tratamento - $3^{\mathrm{a}}$ edição. Porto Alegre: Artmed, 2006.

CAIUBY, A. V. S.; KARAM, C. H. Aspectos Psicológicos de Pacientes com Insuficiência Renal Crônica. In: ISMAEL, S. M. C. (Org), A Prática Psicológica e sua Interface com as Doenças. (p. 131-148) São Paulo, SP: Casa do Psicólogo, 2010.

CAMPOS, C. J. G.; TURATO, E. R. Tratamento Hemodialítico Sob a Ótica do Doente Renal: Estudo Clínico Qualitativo. Revista Brasileira de Enfermagem, 63(5), 2010. p. 799-805. http://www.scielo.br/scielo. php?script=sci_arttext\&pid=S0034-71672010000500017. Acesso em: $20 \mathrm{de}$ ago. 2018.

DINIZ, D. P.; SCHOR, N. Psiconefrologia: Humanização e Qualidade de vida. In: Denise Pará Diniz e Nestor Schor (Coord). Guia de Qualidade de Vida (pp. 35-53). Barueri, SP: Manole, 2006.

FARIAS, L. A. B. A Produção Brasileira Sobre a Atuação do Psicólogo Junto a Pacientes com Insuficiência Renal Crônica em Diálise: Uma Análise Crítica. Dissertação de Mestrado. Pontifícia Universidade Católica de São Paulo, São Paulo, SP, Brasil, 2012. https://tede2.pucsp.br/handle/handle/15111. Acesso em: 03 de out. 2018.

GUYTON, A. C., Hall, J. E. Tratado de Fisiologia Médica. 11. a ed. Rio de Janeiro: Editora Elsevier, 2006.

KÜBLER-ROSS, E. Sobre a Morte e o Morrer. São Paulo, SP: WMF Martins Fontes, 2011.

MACIEL; M. G. S. Modelo de intervenção em cuidados paliativos: a experiência do HSPE-SP. In: PIMENTA, C. A. M.; MOTA; D. D. C. F.; CRUZ, D. A. L. M. (Org.), Dor e Cuidados Paliativos: Enfermagem,

Medicina e Psicologia. (p. 384-408). Barueri: Manole, 2006.

MALDANER, C. R. et. al. Fatores que Influenciam a Adesão ao Tratamento na Doença Renal Crônica: O Doente em Terapia Hemodialítica. Revista Gaúcha de Enfermagem, 29 (4): 647-53, 2008. https://seer.ufrgs.br/ RevistaGauchadeEnfermagem/article/view/7638. Acesso em 20 de ago. 2018.

MATURANA, AP. P.M., CALLEGARI, B., SCHIAVON, V. Atuação do Psicólogo Hospitalar na Insuficiência Renal Crônica. Psicologia Hospitalar 14 (1): 94-116, 2016. http://pepsic.bvsalud.org/scielo.php?script=sci_ arttext\&pid=S1677-74092016000100006. Acesso em: 10 de set. 2018.

MUCCILlO, N. O Preparo do Corpo Após a Morte: Aspectos Culturais, Cuidados Físicos e Emocionais. In: PIMENTA, C. A. M.; MOTA; D. D. C. F.; CRUZ, D. A. L. M. (Org.), Dor e cuidados paliativos: enfermagem, medicina e psicologia (p. 347-359). Barueri: Manole, 2006.

NAKAO, R. T. Variáveis Sociodemográficas, Clínicas e Psicológicas Associadas à Adesão à Hemodiálise. Dissertação de Mestrado. Universidade de São Paulo, Ribeirão Preto, SP, Brasil, 2013. http://www.teses.usp.br/teses/ disponiveis/59/59137/tde-07102013-104339/publico/RenataNakao.pdf. Acesso em: 18 de set. 2018.

NASCIMENTO, F.A.F. Uma Contribuição às Reflexões Sobre os Aspectos Emocionais e o Papel do Psicólogo na Hemodiálise. Rev SBPH. 16(1):70-87, 2013. http://pepsic.bvsalud.org/scielo.php?script=sci_arttext\&pid=S151608582013000100005 . Acesso em: 20 de ago. 2018.

PACHECO, G.S; SANTOS, I; BREGMAN, R. Características de Clientes com Doença Renal Crônica: Evidências para o Ensino do Autocuidado. Revista de enfermagem - UERJ. 2006; 14:434-9. http://www.facenf.uerj.br/ v14n3/v14n3a16.pdf. acesso em: 10 de set. 2018.

REIS, C. K.; GUIRARDELlO, E. B.; CAMPOS, C. J. G. O Indivíduo Renal Crônico e as Demandas de Atenção. Brasília: Revista Brasileira de Enfermagem; 61(3), 2008. pp.336-341. http://www.scielo.br/scielo. php?script $=$ sci_arttext\&pid=S0034-71672008000300010. Acesso em: 05 de out. 2018 .

RESENDE, M. C., SANTOS, F. A., SOUZA, M. M. \& MARQUES, T. P. Atendimento Psicológico a Pacientes com Insuficiência Renal Crônica: Em Busca de Ajustamento Psicológico. Psicologia Clínica, 2007, 19 (2), 87-99. http://www.scielo.br/scielo.php?script=sci_arttext\&pid=S010356652007000200007. Acesso de 05 de out. 2018.
RIELLA, Miguel Carlos et al. Princípios de Nefrologia e Distúrbios Hidroeletrolíticos. 5a edição. Rio de Janeiro: Guanabara Koogan, 2010.

ROMÃO JÚNIOR, J.E. Doença Renal Crônica: Definição, Epidemiologia e Classificação. Jornal Brasileiro de Nefrologia. 2004; 26:1-3. http://www.bjn. org.br/details/1183/pt-BR/doenca-renal-cronica--definicao--epidemiologiae-classificacao. Acesso em: 10 set. 2018.

SILVA, G. L. D. da; THOMÉ, E. G. da R. Complicações do Procedimento Hemodialítico em Pacientes com Insuficiência Renal Aguda: Intervenções de Enfermagem. Revista Gaúcha de Enfermagem 30(1): 33-9. 2009. https:// seer.ufrgs.br/RevistaGauchadeEnfermagem/article/view/3844. Acesso em: 23 set. 2018

SIMONE, M. C. L. Analítica dos Sentidos e Significados Atribuídos por Pessoas Vivendo com Insuficiência Renal Crônica em Tratamento de Hemodiálise: Seus Modos de Ser-no-Mundo/Transcendência. Dissertação de Mestrado. Pontifícia Universidade Católica de São Paulo, São Paulo, SP, Brasil, 2011. https://tede2.pucsp.br/handle/handle/15013. Acesso em: 10 de ago. 2018 .

SOCIEDADE BRASILEIRA DE NEFROLOGIA (2014, 04 de abril). SBN Insuficiência Renal Crônica. Disponível em: https://www.sbn.org.br/. Acesso em: 14 ago. 2018.

SOUSA, M. R. G. et al. Eventos adversos em Hemodiálise: Relatos de Profissionais de Enfermagem. Revista da Escola de Enfermagem USP 47(1): 76-83. 2013. http://www.scielo.br/scielo.php?script=sci arttext\&pid=S0080-62342013000100010. Acesso em: 10 de ago. 2018.

STRAUB, R. O. Psicologia da Saúde: Uma Abordagem Psicossocial. (3a ed.). Porto Alegre: Artmed, 2014.

TORRES, T. L. O Psicólogo Centrado na Pessoa e a Instituição Hospitalar. In: Fórum Brasileiro da A.C.P. Ouro Preto, 1999. Disponível em: https:// apacp.org.br/diversos/artigos/. Acesso em: 05 out. 2018. 